\title{
АНАЛИЗ СУЩЕСТВУЮЩИХ ФОРМАЛЬНЫХ ПОДХОДОВ К ОПРЕДЕЛЕНИЮ ПОНЯТИЯ «СЛОЖНАЯ ЗАДАЧА»
}

\section{ANALYSIS OF EXISTING FORMAL APPROACHES TO THE DEFINITION OF THE CONCEPT «THE COMPLEXITY OF THE TASK»}

\section{E. Trofimets V. Trofimets}

Summary. The article analyzes the definitions of the concepts of "complex task». Two types of tasks are considered. The situation of solving a complex problem is given. A hypothetical decision support system is considered, which includes knowledge from the field of mathematics for calculating the areas of triangles.

Keywords: complex task, goal, conditions, canonical form, decision support system.
Трофимец Елена Николаевна К.n.н., дочент, ФГБОУ ВО «Санкт-Петербургский университет Государственной противопожарной службы Министерства РФ по делам гражданской обороны, чрезвычайным ситуациям и ликвидации последствий стихийных бедствий» ezemifort@inbox.ru

трофимец Валерий Ярославович Д.т.н., профессор, Санкт-Петербургский горный университет zemifort@inbox.ru

Аннотация. В статье проанализированы определения понятий «сложная задача». Рассмотрены два типа задач. Приведена ситуация по решению сложной задачи. Рассмотрена гипотетическая система поддержки принятия решений, в которую заложены знания из области математики по расчету площадей треугольников.

Ключевые слова: сложная задача, цель, условия, каноническая форма, система поддержки принятия решений.
$\Pi$ од задачей понимается вопрос, требующий решения на основании определённых знаний и размышлений.

Каноническая форма задачи представляет собой логическое высказывание вида:

«Дано V, требуется W» (краткая запись < V, W >), где V - условия задачи; W - цель задачи.

Цель задачи может быть сформулирована двояко, исходя из чего различают два типа задач: задачи «на нахождение» и задачи «на доказательство» [1].

Целью (результатом решения) задач первого типа является найденный в процессе решения неизвестный объект (состояние), удовлетворяющий условиям задачи. Этот объект (состояние) может иметь самую различную физическую природу: материальную, временную, энергетическую, информационную.

Целью (результатом решения) задач второго типа является последовательность умозаключений, позволяющая доказать по установленным правилам истин- ность или ложность следования из исходных посылок установленного заключения. Таким образом, в задачах «на доказательство» объект (состояние) задан в виде заключения, т.е. известен заранее.

Условия задачи $V$ можно представить в виде объединения двух непересекающихся подмножеств:

$$
V=X \cup Z
$$

где $X$ - множество переменных, значения которых задаются и являются исходными данными для решения задачи;

$Z$ - множество знаний, которыми обладает эксперт-аналитик по решению определённой задачи.

Тогда, учитывая (2), каноническая форма задачи может быть трансформирована в логическое высказывание следующего вида:

«Имея $Z$, найти $W$ по $X$ ».

В общем случае под знаниями $Z$ понимается информация, которая отражает закономерности, существую- 
щие в выбранной предметной области и позволяющие устанавливать новые факты (выводить новые знания), не зафиксированные в ней на данный момент времени. Характерными особенностями знаний, отличающими их от данных, являются связь знаний с понятием интенсионала (данные задаются только экстенсионально) и представление знаний в процедурной форме или возможность их преобразования в такую форму [2-9].

Программные средства системы поддержки принятия решений (СППР) фактически являются одним из способов представления знаний о методах решения аналитических задач в конкретных предметных областях.

Пусть $Z^{*}$ — это множество знаний, необходимых для решения определённой задачи. Возможны три варианта отношений между $Z$ и $Z^{*}$ :
1) $Z \subset Z^{*}$;
2) $Z=Z^{*}$;
3) $Z \supset Z^{*}$.

Первый вариант характеризуется недостаточностью знаний для решения задачи. Для нахождения решения необходимо такое приращение знаний $\Delta Z$, чтобы выполнялось условие:

$$
Z \cup \Delta Z \supseteq Z^{*} \text {. (5) }
$$

В СППР приращение $\Delta Z$ может быть достигнуто или путем подключения дополнительных программных модулей (для СППР 1-го и 2-го классов), или в режиме обучения и самообучения (для СППР 3-го класса).

Второй и третий варианты отношений между $Z$ и $Z^{*}$ характеризуются соответственно достаточностью и избыточностью знаний для решения определённой задачи. Для СППР особый интерес представляет третий вариант с избыточностью знаний.

Первоначально понятие избыточности использовалось в теории информации применительно к передаче сигналов по каналам связи.

В работе [3] К. Шеннон определил избыточность как меру неэкономности кодирования. Включение лишних символов ведёт, с одной стороны, к увеличению времени передачи сообщений, а с другой - к повышению достоверности передачи.

Для СППР введение избыточных знаний также имеет как положительные, так и отрицательные стороны. Необходимо отметить, что в данном контексте под избыточными понимаются не знания-копии, полученные путём тиражирования знаний-оригиналов с целью повышения надёжности их хранения. Здесь под избыточными понимаются знания, имеющие другую смысловую форму представления (другое семантическое наполнение), но ведущие к получению того же результата, что и базовые знания.

В качестве примера может быть рассмотрена гипотетическая СППР, в которую заложены знания из области математики по расчету площадей треугольников. Пусть в качестве базовых знаний в систему заложены следующие формулы, позволяющие вычислять площадь треугольника в зависимости от поступающей входной информации:

$$
\begin{aligned}
& S=0,5 \times a \times b \times \sin \gamma, \\
& S=\sqrt{p(p-a)(p-b)(p-c}, \\
& S=0,5 \times a \times h, \\
& S=\frac{0,5 \times a^{2} \times \sin \alpha \times \sin \gamma}{\sin \beta} .
\end{aligned}
$$

Введение в систему формул для нахождения

$$
\begin{aligned}
& \text { площадей равностороннего } S=0,25 \times a \times \sqrt{3} \text {, } \\
& \text { равнобедренного } S=0,5 \times a \times \sqrt{b^{2}-\frac{a^{2}}{4}}, \\
& \text { прямоугольного } S=0,5 \times a \times b
\end{aligned}
$$

треугольников приводит к избыточности, т.к. они являются частным случаем базовых формул и не требуют обязательного знания при расчёте площадей. Кроме того, их введение требует дополнительных объёмов памяти системы, что для задач большой размерности может представлять значительную величину. Однако если на вход системы поступает информация, что треугольник имеет определенный вид (равносторонний, равнобедренный, прямоугольный), то целесообразно применение частных формул, более эффективных с вычислительной точки зрения (например, при расчете площади прямоугольного треугольника частная формула в 3,5-14 раз эффективней основной формулы в зависимости от типа, формата переменных и использования режима сопроцессора (см. табл. 1).

Приведенный пример гипотетической СППР упрощенно моделирует реальную ситуацию решения сложной задачи, когда поступление дополнительной информации снижает неопределенность исходных данных, что может значительно облегчить (ускорить) решение задачи. При этом следует, конечно, сделать оговорку о достоверности поступившей информации. Если информация каким-либо образом (случайным или пред- 
Таблица 1.

\begin{tabular}{|c|c|c|c|c|}
\hline $\begin{array}{l}\text { Тип } \\
\text { переменных }\end{array}$ & $\begin{array}{l}\text { Число } \\
\text { итераций }\end{array}$ & $\begin{array}{l}\text { Время } \\
\text { исполнения } \\
\text { первого цикла } \\
T 1, \mathrm{~ms}\end{array}$ & $\begin{array}{l}\text { Время } \\
\text { исполнения } \\
\text { второго цикла } \\
T 2, \mathrm{~ms}\end{array}$ & $\begin{array}{l}\text { Отношение } \\
T 1 / T 2\end{array}$ \\
\hline \multicolumn{5}{|c|}{ Режим 1 (без сопроцессора) } \\
\hline $\begin{array}{l}a: \text { int } \\
b: \text { int }\end{array}$ & $\begin{array}{l}10000 \\
100000 \\
1000000\end{array}$ & $\begin{array}{l}11 \\
110 \\
1087\end{array}$ & $\begin{array}{l}154 \\
1539 \\
15330\end{array}$ & $\begin{array}{l}14,00 \\
13,94 \\
14,10\end{array}$ \\
\hline $\begin{array}{l}a \text { : real } \\
b: \text { int }\end{array}$ & $\begin{array}{l}10000 \\
100000 \\
1000000\end{array}$ & $\begin{array}{l}11 \\
126 \\
1203\end{array}$ & $\begin{array}{l}159 \\
1604 \\
16049\end{array}$ & $\begin{array}{l}14,45 \\
12,73 \\
13,34\end{array}$ \\
\hline $\begin{array}{l}a: \text { real } \\
b: \text { real }\end{array}$ & $\begin{array}{l}10000 \\
100000 \\
1000000\end{array}$ & $\begin{array}{l}17 \\
182 \\
1764\end{array}$ & $\begin{array}{l}165 \\
1658 \\
16689\end{array}$ & $\begin{array}{l}9,71 \\
9,11 \\
9,42\end{array}$ \\
\hline \multicolumn{5}{|c|}{ Режим 2 (с сопроцессором) } \\
\hline $\begin{array}{l}a: \text { int } \\
b: \text { int }\end{array}$ & $\begin{array}{l}10000 \\
100000 \\
1000000\end{array}$ & \begin{tabular}{|l}
5 \\
82 \\
791 \\
\end{tabular} & $\begin{array}{l}33 \\
280 \\
2823\end{array}$ & $\begin{array}{l}6,60 \\
3,41 \\
3,57\end{array}$ \\
\hline $\begin{array}{l}a: \text { real } \\
b: \text { int }\end{array}$ & $\begin{array}{l}10000 \\
100000 \\
1000000 \\
\end{array}$ & $\begin{array}{l}5 \\
83 \\
791 \\
\end{array}$ & $\begin{array}{l}33 \\
280 \\
2823 \\
\end{array}$ & $\begin{array}{l}6,60 \\
3,37 \\
3,57 \\
\end{array}$ \\
\hline $\begin{array}{l}a: \text { real } \\
b: \text { real }\end{array}$ & $\begin{array}{l}10000 \\
100000 \\
1000000\end{array}$ & $\begin{array}{l}5 \\
83 \\
791\end{array}$ & $\begin{array}{l}33 \\
280 \\
2823\end{array}$ & $\begin{array}{l}6,60 \\
3,37 \\
3,57\end{array}$ \\
\hline
\end{tabular}

намеренным) будет искажена, то это только затруднит решение задачи.

Кроме того, введение в СППР избыточных модулей отражается и на её свойстве доступности пользователю. Например, наличие в системе нескольких статистических пакетов (STADIA, ME3O3ABP, STATISTICA, STATGRAPHICS, SPSS и т.п.) позволяет эксперту-аналитику выбрать для работы тот из них, который для него наиболее приемлем (по функциональным возможностям, интерфейсу, удобству работы и др.). Конечно, этот аспект избыточности СППР требует дополнительных финансовых вложений, поэтому на практике он не получил широкого распространения.

Таким образом, целесообразность введения в СППР избыточных знаний необходимо оценивать с учётом вероятности появления на входе системы информации, позволяющей использовать избыточные знания для повышения качества процесса нахождения решения. Кроме того, введение в СППР избыточных знаний создаёт предпосылки для её дальнейшего развития и совершенствования. Так, в работе [4] Дж. фон Нейман связывал избыточность знаний с возможностью переработки информации большой сложности, т.е. с функциональной сложностью системы. Под функциональной сложностью Нейман понимал «способность системы делать нечто существенное». В [5] под функциональной сложностью системы понимается максимальная сложность задачи или пакета задач, которую ещё способна решить система при неизменной сложности её структуры за заданное время:

$$
C \pi_{\text {cuсm }}=\max C \pi_{3 а д} \mid \text { Слстр }=\text { const }, t_{\text {реш }} \leq t_{\text {задан }}
$$

где $C \pi_{\text {сист }}-$ функциональная сложность системы; max $C л_{\text {зад }}$ - максимальная сложность задачи (пакета задач);

$C л_{c m p}$ - сложность структуры системы;

$t_{\text {реш }}$ - время решения задачи (пакета задач);

$t_{\text {задан }}$ - заданное время решения задачи (пакета задач).

В этом случае возникает необходимость в определении понятия «сложная задача» и в нахождении количественной меры сложности задач.

Попытки дать строгое определение понятию «сложная задача» связаны со значительными трудностями, так как данное понятие имеет субъективную обусловленность и связано со способностью конкретного индивида понять и решить задачу. Этим объясняется тот факт, что понятие «сложная задача» до настоящего времени остаётся в значительной степени интуитивным. 


\section{ЛИТЕРАТУРА}

1. Пойа Г. Математическое открытие.-М.: Наука, 1976.

2. Поспелов Д. А. Ситуационное управление.-М.: Наука, 1986.

3. Шеннон К. Работы по теории информации и кибернетике.-М.: Изд-во иностр. лит., 1963.

4. Нейман Дж. фон. Теория самовоспроизводящихся автоматов.-М.: Мир, 1971.

5. Клир Дж. Системология: автоматизация решения системных задач. - М.: Радио и связь, 1990.

6. Математическое и программное обеспечение информационных систем поддержки принятия решений: монография / Трофимец Е. Н.—— Санкт-Петербург: Санкт-Петербургский университет ГПС МЧС России, 2019. - 176 с.

7. Трофимец Е.Н., Трофимец А. А. Анализ содержания информационно-аналитической подготовки специалистов экономического профиля // Материалы конференции «Технические и естественные науки; Безопасность: информация, техника, управление». Санкт-Петербург, 24-28 февраля 2021 года. ГНИИ НАЦРАЗИТИЕ. Сборник избранных статей по материалам научных конференций ГНИИ «Нацразвитие». Санкт-Петербург, 2021. С. 94-96.2021.

8. Трофимец Е.Н., Трофимец А. А. Об использовании взаимосвязанных элементов математического аппарата и экономических понятий // Материалы конференции «Научные исследования в современном мире. Теория и практика; фундаментальные и прикладные исследования. Актуальные проблемы и достижения; современные методы и инновации в науке; исследование и практика в социально-экономической и гуманитарной сфере». Санкт-Петербург, 10-13 февраля 2021 года. ГНИИ НАЦРАЗИТИЕ. Сборник избранных статей по материалам научных конференций ГНИИ «Нацразвитие». Санкт-Петербург, 2021. С. 139-142. 2021.

9. Трофимец А.А., Трофимец Е. Н. Программные продукты как составная часть информационно-аналитической подготовки специалистов экономического профиля // Журнал «Актуальные научные исследования в современном мире». Общественная организация «Институт социальной трансформации» (Переяслав-Хмельницкий), 2021. № 5-7 (73). С. 143-147. 2021.

( ) Трофимец Елена Николаевна ( ezemifort@inbox.ru ), Трофимец Валерий Ярославович ( zemifort@inbox.ru ).

Журнал «Современная наука: актуальные проблемы теории и практики»

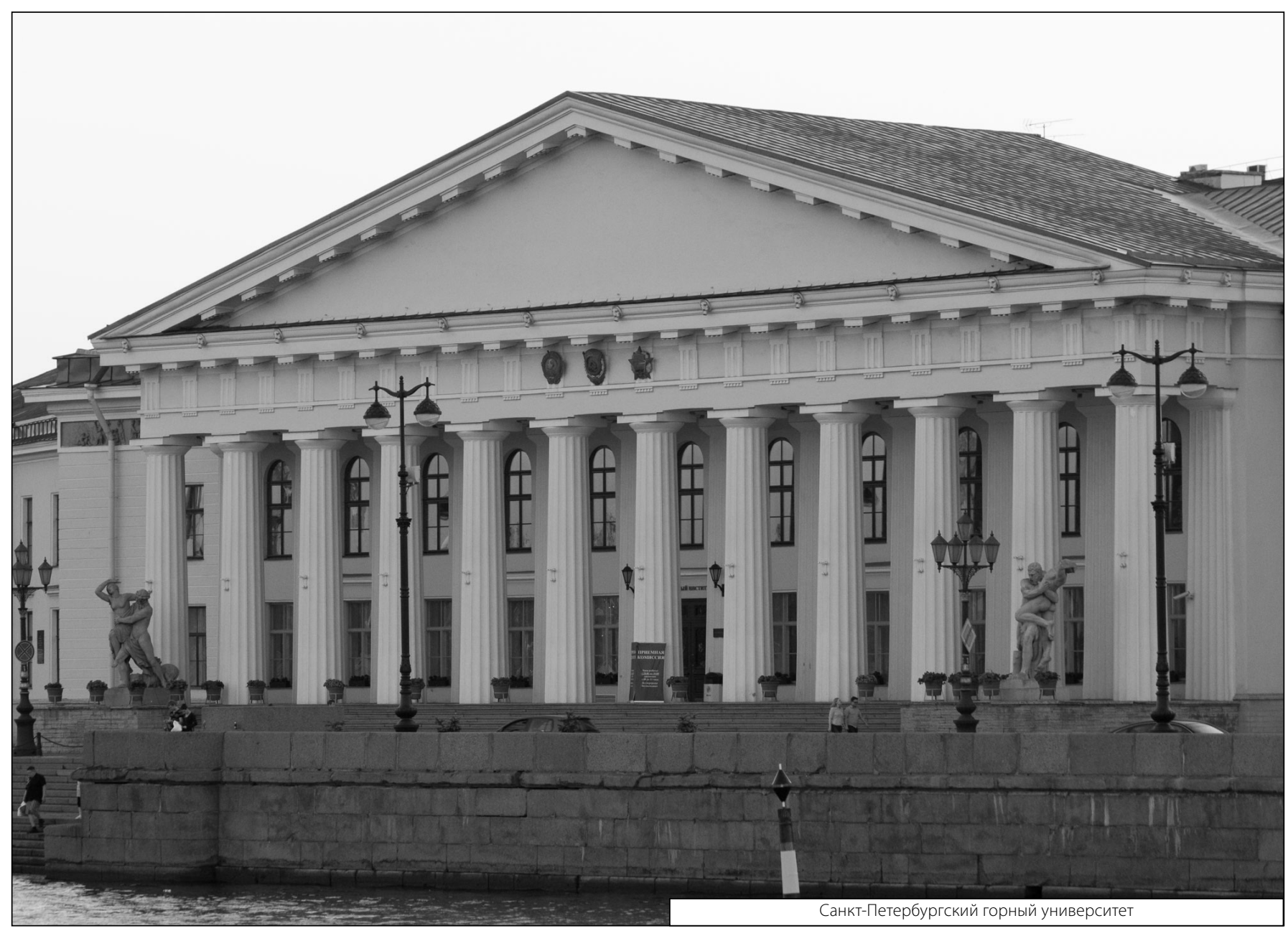

